\title{
Effect of habitual reading direction on saccadic eye movements
}

A Lyu ${ }^{\mathrm{a}, \mathrm{b}}(\mathrm{BSc}, \mathrm{MSc}), \mathrm{L}$ Abel b,c $(\mathrm{PhD})$, AMY Cheong ${ }^{\mathrm{a}}(\mathrm{PhD}, \mathrm{BSc}($ Hons))

a School of Optometry, The Hong Kong Polytechnic University, Hong Kong

${ }^{\mathrm{b}}$ Department of Optometry and Vision Sciences, University of Melbourne, Australia

c School of Medicine, Faculty of Health, Deakin University, Australia

Correspondence's Address: Allen MY Cheong

School of Optometry, The Hong Kong Polytechnic University, Hung Hom, Hong Kong

Telephone:

$852-2766-6108$

Facsimile:

$852-2764-6051$

Electronic mail:

allen.my.cheong@polyu.edu.hk

Number of tables:

2

Number of figures:

4

Journal submission: Plos One

Date of $1^{\text {st }}$ submission: $\quad 10^{\text {th }}$ Jan 2022

Contributions of authors to the article:

- Anqi Lyu: Experimental design, data collection, analysis and manuscript writing.

- Larry Abel and Allen Cheong are supervisors of Anqi Lyu (postgraduate student). We involved in experimental design, data analysis and manuscript writing.

Email correspondence of authors:

- Lyu A:

an-qi.lyu@connect.polyu.hk

- Abel L:

larry.abel@deakin.edu.au

- Cheong AMY:

allen.my.cheong@polyu.edu.hk 


\section{Abstract}

Cognitive processes can influence the characteristics of saccadic eye movements. Reading habits, including habitual reading direction, also affects cognitive and visuospatial processes, favouring attention to the side where reading begins. Few studies have investigated the effect of habitual reading direction on saccade directionality of lowcognitive-demand stimuli (such as dots). The current study examined horizontal prosaccade, antisaccade and self-paced saccade in subjects with two primary habitual reading directions. We hypothesised that saccades responding to the target in subject's habitual reading direction would show a longer prosaccade latency and lower antisaccade error rate (errors being a reflexive glance to a sudden-appearing target, rather than a saccade away from it). Sixteen young Chinese participants with primary habitual reading direction from left to right and sixteen young Arabic and Persian participants with primary habitual reading direction from right to left were recruited. Subjects needed to look towards a $5^{\circ} / 10^{\circ}$ target in the prosaccade task or look towards the mirror image location of the target in the antisaccade task and look between two 10-degree targets in the self-paced saccade task. Only Arabic and Persian participants showed a shorter and directional prosaccade latency towards $5^{\circ}$ target against their habitual reading direction. No significant effect of primary reading direction on antisaccade latency towards the correct directions was found. However, we found that Chinese readers generated significantly shorter prosaccade latencies and higher antisaccade directional errors compared with Arabic and Persian readers. The present study provides an insight into the effect of reading habits on saccadic eye movements in response to lowcognitive-demand stimuli and offers a platform for future studies to investigate the relationship between reading habits and neural mechanisms of eye movement behaviours. 


\section{1. Introduction}

\section{$2 \quad 1.1$ Saccadic eye movements}

3

4

Humans do not look at a scene with steady gaze. Our eyes move around, bringing the interesting parts of the scene to the fovea with the frequency of 2 or 3 fixations per second [1]. In fact, saccadic eye movement is one of the fastest movements produced by the human body, serving in bringing the images of objects of interest into central vision for detailed analysis. The perception of the environment relies on saccades and fixations which are the stops in-between saccades [2]. A distributed network including cortical (mainly frontal and parietal) and subcortical (basal ganglion, superior colliculus, midbrain, brain stem, thalamus and cerebellum) areas are involved in generating saccades [3]. It has been suggested that understanding the saccadic system provides researchers a valuable "microcosm of the brain" as its input can be controlled and manipulated, while its output can be accurately recorded and quantified using different experimental paradigms [4]. A range of eye movement tasks have been used in the literature to examine the characteristics of saccades, including prosaccade, antisaccade and self-paced saccade tasks. Prosaccades, which are also known as reflexive saccades, test the response time (latency) and accuracy of a saccade (saccade gain in terms of the ratio of saccade amplitude / target amplitude) to a sudden-onset peripheral visual stimulus. An antisaccade requires the suppression of a reflexive saccade towards a suddenonset stimulus and the execution of a voluntary saccade to the opposite direction of the stimulus. The parallel nature of antisaccade programming assumes a competition arises between the exogenously triggered prosaccade and the endogenously initiated antisaccade at the onset of stimulus [5-7]. For example, if the exogenously triggered prosaccade is programmed too fast (or the endogenously initiated antisaccde is too slow to reach the threshold for activation), it "wins" the competition and make a reflexive saccade first (i.e., 
antisaccade directional error), followed by a corrective antisaccade [8]. Directional error rate (i.e., the proportion of glances towards the stimulus) and the latency of correct response are commonly analysed in antisaccade task. Self-paced saccade task has been considered as an almost entirely volitional eye movement task that requires repetitive and self-initiated refixations between two static visual stimuli [9]. neurological and psychiatric disorders [10], such as schizophrenia [11], attention-deficit hyperactivity disorder (ADHD) [12-14], Parkinson's disease [3, 15, 16] and depression [17]. In particular, a vast array of studies have suggested that many cognitive processes, including those involved in attention [18-20], working memory [21] and learning [22], have an impact on the characteristics of saccadic eye movements.

\subsection{Effect of cognitive process on saccadic eye movement}

Attention is needed to orient the target location prior to the execution of a saccade [20]. Saslow reported a decrease in prosaccade latency from $200 \mathrm{msec}$ to $150 \mathrm{msec}$ if the stimulus appeared $200 \mathrm{msec}$ or longer after the termination of the fixation, compared to the situation where the offset of fixation and onset of stimulation occurred simultaneously [23]. By introducing a medium temporal gap $(200-250 \mathrm{msec})$ between the offset of a central fixation target and the onset of a peripheral stimulus, Fischer and Weber found a significant decrease in antisaccade latency but a significant increase in antisaccade error rate [18]. One explanation for these changes in saccades was that this temporal gap contributed to the disengagement of attention before the target appeared. Moreover, studies manipulating the likelihood of the target presenting on either left or right side of a central fixation point found that subjects showed shorter prosaccade latency to the target direction with a higher probability of presentation. This suggested an effect of learning in modifying the prosaccade performance $[22,24]$. 
While changing the direction of letters and words within English sentences (i.e., both

letters and words were orientated from right to left), Inhoff et al. reported less efficient

texts. However, these performances improved with practice [25]. In addition to these studies,

extensive findings have revealed a wide range of cognitive processes influencing saccadic

eye movements [18-20]. Even a simple prosaccade involves a complex weighting of both

bottom-up information (stimulus properties) and top-down information (cognitive factors),

although the precise nature for the degrees of the control remains unclear [8]. In addition, our

cognitive systems are shaped or influenced by cultural practices such as reading habits (see

below of section 1.3), which suggests the impact of reading habits on characteristics of saccadic eye movements.

\subsection{Effect of habitual reading direction on cognitive systems} processes [27]. Reading direction has been found to influence many cognitive functions, such as directional differences in facial expression perception [28], aesthetic preference [29] and utilization of visual space [30]. Especially, visuospatial attention can be modulated by the habitual reading direction. During a letter matching task, English readers with habitual reading direction of left-to-right (LTR) spent a longer time in responding to the stimulus in the right visual field, while Hebrew readers with habitual reading direction of right-to-left (RTL) took longer to respond to the stimulus that appeared in the left visual field. They 
direction on the asymmetries of visuospatial attention [32-34]. For example, Rinaldi and colleagues (2014) compared the performance on a star cancellation task between Italian and Israeli subjects who were instructed to mark the small stars amongst many randomly distributed distractors (large stars, English or Hebrew letters and words). They found that monolingual Italian subjects (i.e., reading from LTR) made more omissions in the right visual field, while monolingual Israeli subjects (i.e., reading from RTL) omitted more targets in the left visual field [33]. However, bilingual subjects who managed reading in both directions did not show any spatial asymmetries. Further, Afsari and colleagues (2016) examined the effect of habitual reading direction in bilingual readers of a native LTR language and a secondary RTL language. They found that native LTR readers who studied a secondary RTL language in late life showed a leftward bias with more fixations on the left part of a natural image, and this horizontal bias of the exploration of images did not alter when they first read either LTR or RTL text primes [34].

In addition to the biased visuospatial attention, we questioned whether reading direction also contributed to the left-right asymmetry of saccadic eye movements. While

90 reading continuous text, LTR texts such as English [35] and German [36] elicit saccades towards the location slightly to the left of a word centre, while RTL scripts such as Hebrew [37] and Uighur [38] have saccades landing to the position slightly to the right of a word centre. In addition to saccades generated towards to the left and right directions, Yan et al. took the advantage that Chinese text can be orientated horizontally and vertically without disturbing the shape of characters and reported a similar saccadic landing position for 28 young readers reading horizontal and vertical Chinese texts [39]. These studies demonstrated

97 that reading direction affected saccadic eye movements during high-level reading processes. Nevertheless, fewer studies have investigated the impact of the habitual reading direction on the directionality of saccadic eye movements during low-cognitively demanded tasks such as 
responding to a dot. Most of the studies investigating the left-right asymmetry of these cognitive-demand stimuli would help researchers to better investigate the differences of eye movement control across populations. reflexively and volitionally initiated saccades. We hypothesized that readers who habitually read from LTR should show a leftward asymmetry in the saccadic parameters (i.e., shorter

110 prosaccade latency and higher antisaccade error rate) when they made a saccade responding

111 to the target appeared in the direction where reading began (i.e., the target appeared at the left

112 of a fixation point) compared with the target appeared along their habitual reading direction

113 (i.e., the target appeared at the right of the fixation point). In contrast, readers who habitually

114 read from RTL would show a rightward asymmetry.

\section{2. Material and methods}

\section{$116 \quad 2.1 \quad$ Subjects}

32 young university students who were bilingual speakers and readers were recruited

118 from the University of Melbourne (20) and The Hong Kong Polytechnic University (16). The

119 calculated sample size provided $85 \%$ power to detect a significant difference (an estimated

120 effect size of 0.71 ) between the 2 reading directions of $5^{\circ}$ stimulus at the one-tailed 0.05

121 alpha level. 16 subjects were Chinese readers whose primary reading direction was LTR and

12216 Arabic and Persian readers (12 Arabic and 4 Persian) whose primary reading direction was RTL. All participants were aged between 18 and 35 with normal or corrected to normal 
124 vision and started to learn English (with reading direction of LTR) since their early

125 childhoods. To control the potential confounding influence of education level, this factor was

126 controlled and matched between these 2 groups. Participants in the RTL group were

127 significantly older than the LTR group. Nevertheless, horizontal saccade latency is relatively

128 stable from age of 14 to 50 years old [45], so this factor should not be a concern. Exclusion

129 criteria were any history of ophthalmic, neurological or psychotic illness, or any medications

130 intake that might affect eye movements. Subjects were separated into 2 groups according to

131 their primary habitual reading direction. The characteristics of the participants are shown in

132 Table 1. Informed consent was obtained in accordance with a protocol approved by the

133 University of Melbourne human research ethics committee (HREC \#1647981.1) and

134 Department of Research Committee of the School of Optometry of The Hong Kong

135 Polytechnic University (HSEARS20191217001). The study followed the tenets of the

136 Declaration of Helsinki.

137 Table $1 \quad$ Descriptive characteristics of participants

\begin{tabular}{c|ccc}
\hline \hline & $\begin{array}{c}\text { Chinese participants } \\
(\mathrm{N}=16)\end{array}$ & $\begin{array}{c}\text { Arabic }(\mathrm{N}=12) \text { and Persian } \\
(\mathrm{N}=4) \text { participants }\end{array}$ & P-value \\
\hline $\begin{array}{c}\text { Habitual reading } \\
\text { direction }\end{array}$ & From left to right (LTR) & From right to left (RTL) & \\
$\begin{array}{c}\text { Age (y) } \\
\text { mean (SD) }\end{array}$ & $23.8(3.4)$ & $27.1(3.8)$ & 0.02 \\
$\begin{array}{c}\text { range (y) } \\
\begin{array}{c}\text { English education } \\
(\mathrm{y}), \text { mean (SD) }\end{array}\end{array}$ & $19-32$ & $18-34$ & \\
range (y) & $16.8(3.3)$ & $16.6(6.4)$ & 0.89 \\
\hline \hline
\end{tabular}

\subsection{Apparatus and stimuli}

As the data were collected at 2 sites, minor differences of experimental setting were

141 present, including presenting monitor and testing distance, whereas the stimulus size and 
142 distance were adjusted so that same visual angle was elicited. Both the testing sequence and

143 program (including resolution and refresh rate) were identical between the 2 sites. The

144 stimulus was a 1-degree black dot against a white background on a 27-inch (U2711B, Dell

145 Technologies, Round Rock, Texas, United States) or a 24-inch LCD monitor (BENQ x12540)

146 in Melbourne and Hong Kong site respectively. Participants sat comfortably at $75 \mathrm{~cm}$

147 (Melbourne) or $65 \mathrm{~cm}$ (Hong Kong) in front of the monitor with chin resting on a chinrest to

148 stabilize their head position. Movement of both eyes was recorded using an infrared video

149 eye tracking system (Eyelink 1000 or Eyelink Portable Duo, SR Research, Scarborough,

150 ONT, Canada) with a sampling rate of $500 \mathrm{~Hz}$. The resolution and refresh rate of the

151 monitors were 1920 x 1080 pixels and $60 \mathrm{~Hz}$. Subjects were asked to perform the following

152 eye movement tasks.

\section{$153 \quad 2.3$ Procedures}

Participants' eye movements were assessed while conducting 3 visual tasks: 1)

prosaccade, 2) antisaccade and 3) self-paced saccade tasks.

Targets were presented pseudorandomly in locations 5 or 10 degrees to the left or

157 right of the centre of the monitor. Participants were instructed to fixate at a centre cross and

158 then to look towards the target in the prosaccade task (see Fig. 1a) or look towards the mirror image of the target in the antisaccade task (see Fig. 1b) as soon as the target was presented and fixation disappeared. Fifty-two trials were conducted in the prosaccade task to assess the

161 prosaccade latency (i.e., reaction time responding to the onset of stimulus) and gain (i.e., ratio

162 of saccadic amplitude to target amplitude). Express saccades whose latency falls between 80

163 to $120 \mathrm{msec}$ [46-48] were excluded from the analysis. Less than $15 \%$ of the trials were

164 excluded for all participants. Fifty-two trials were conducted in the antisaccade task to assess the antisaccade latency of correct responses (i.e., saccades made to the correct direction) and 
were shown for 45 seconds at 10 degrees left and right of the centre of the monitor. saccade and target amplitude) and inter-saccadic intervals (i.e., interval between onset of the saccades) were collected and submitted to data analysis.

\section{Fig. 1 Sample trial of prosaccade and antisaccade task}

Fig. 1a. A sample trial of prosaccade task

when participants need to make a saccade

towards the target as quickly as possible.
Fig. 1b. A sample trial of antisaccade task when participants need to look at the mirror image of the target location as quickly as possible.

\section{$173 \quad 2.4 \quad$ Data analysis}

All statistical analysis was performed using GraphPad Prism version 9.2.0.332 for

175 Windows (GraphPad Software, San Diego, California USA, www.graphpad.com). Eye movement parameters were not significantly different from normal distribution

177 (Kolmogorov-Smirnov goodness of fit test, $\mathrm{p}>0.05$ ). Dependent variables (prosaccade

178 latency, prosaccade gain, correct antisaccade latency, antisaccade error rate, inter-saccadic interval, and gain in self-paced saccades) were analysed using analysis of variance (ANOVA) with group (LTR (Chinese participants) vs. RTL (Arabic and Persian participants)) as

181 between-subject factors and the direction of stimulus (with- vs. against habitual reading

182 direction $)$ and / or the magnitude of stimulus from the fixation $\left(5^{\circ}\right.$ vs. $\left.10^{\circ}\right)$ as within-subject

183 factors, to assess any significant effect or interaction. A p-value of less than 0.05 was considered statistically significant. 


\section{3. Results}

\section{$186 \quad 3.1 \quad$ Effects of habitual reading direction on prosaccade eye}

\section{movements}

The average prosaccade latency of the RTL group in response to a sudden-onset stimulus was significantly longer than that of the LTR group (mean 188.06 vs. $174.41 \mathrm{msec}$,

$190 \mathrm{~F}(1,60)=5.61, \mathrm{p}=0.02)$. Bonferroni's post-hoc analysis demonstrated that RTL participants required longer prosaccade latency in responding to targets presented at $5^{\circ}$ along their habitual reading direction compared with LTR participants (198.98 vs. $167.75 \mathrm{msec}, \mathrm{p}=0.01$ ). Neither stimulus direction (i.e., presented with- or against-reading direction) or magnitude (i.e., presented at $5^{\circ}$ or $10^{\circ}$ away from fixation) had a significant effect on prosaccade latency $(F(1,60)<1.59, p>0.21)$, whereas a significant interaction between group and stimulus direction was observed $(\mathrm{F}(1,60)=8.07, \mathrm{p}=0.006)$. The RTL participants had longer prosaccade latency when the target was presented $5^{\circ}$ along their habitual reading direction (i.e., target at the left side of the fixation) compared with that appeared against the reading direction (198.98 vs. $178.77 \mathrm{msec}, \mathrm{p}=0.03$ ). However, LTR participants showed similar reaction time for the target appearing towards the two directions (167.75 vs. $176.56 \mathrm{msec}$, $\mathrm{p}>0.99$; Table 2).

Prosaccade gain examines the accuracy of the landing position of prosaccade. Neither

203 of the three independent variables (i.e., group, stimulus direction and stimulus magnitude)

204 significantly affected the gain $(F(1,60)<2.15$, $\mathrm{p}>0.15)$. Nevertheless, similar to the latency result, a significant interaction between group and stimulus direction was found $(F(1,60)=6.93, p=0.01)$. Although no significance was found in the post-hoc analysis (Table 
Table 2 Eye movement characteristics in the prosaccade and antisaccade task (mean and standard deviation)

\begin{tabular}{|c|c|c|c|c|c|c|}
\hline Saccade type & & & & $\begin{array}{l}\text { Chinese participants } \\
\quad(N=16)(L T R)\end{array}$ & $\begin{array}{c}\text { Arabic }(N=12) \text { and Persian } \\
(N=4) \text { participants }(R T L)\end{array}$ & $P$-value \\
\hline \multirow{13}{*}{ Prosaccade } & & Stimulus magnitude & Stimulus direction & & & \\
\hline & \multirow{6}{*}{$\begin{array}{c}\text { Prosaccade } \\
\text { latency } \\
\text { (msec) }\end{array}$} & \multirow{2}{*}{$5^{\circ}$} & With-direction & $167.75(22.06)$ & $198.98(31.76)$ & 0.01* \\
\hline & & & Against-direction & $176.56(24.64)$ & $178.77(23.86)$ & $>0.99$ \\
\hline & & \multicolumn{2}{|c|}{$P$-value } & $>0.99$ & 0.03* & \\
\hline & & \multirow{2}{*}{$10^{\circ}$} & With-direction & $176.00(17.91)$ & $190.33(29.32)$ & $>0.99$ \\
\hline & & & Against-direction & $177.36(36.82)$ & $184.15(19.11)$ & $>0.99$ \\
\hline & & \multicolumn{2}{|c|}{$P$-value } & $>0.99$ & $>0.99$ & \\
\hline & \multirow{6}{*}{$\begin{array}{l}\text { Prosaccade } \\
\quad \text { gain }\end{array}$} & \multirow{2}{*}{$5^{\circ}$} & With-direction & $1.02(0.12)$ & $0.96(0.10)$ & $>0.99$ \\
\hline & & & Against-direction & $0.98(0.08)$ & $1.03(0.23)$ & $>0.99$ \\
\hline & & \multicolumn{2}{|c|}{ P-value } & $>0.99$ & 0.16 & \\
\hline & & \multirow{2}{*}{$10^{\circ}$} & With-direction & $0.99(0.08)$ & $0.94(0.08)$ & $>0.99$ \\
\hline & & & Against-direction & $0.97(0.05)$ & $0.95(0.11)$ & $>0.99$ \\
\hline & & \multicolumn{2}{|c|}{ P-value } & $>0.99$ & $>0.99$ & \\
\hline \multirow{6}{*}{ Antisaccade } & \multirow{6}{*}{$\begin{array}{c}\text { Antisaccade } \\
\text { latency } \\
\text { (msec) }\end{array}$} & \multirow{3}{*}{$5^{\circ}$} & With-direction & $285.73(45.18)$ & $277.74(46.70)$ & $>0.99$ \\
\hline & & & Against-direction & $287.11(38.79)$ & $276.90(45.08)$ & $>0.99$ \\
\hline & & & & $>0.99$ & $>0.99$ & \\
\hline & & \multirow{3}{*}{$10^{\circ}$} & With-direction & $285.96(30.69)$ & $273.05(51.56)$ & $>0.99$ \\
\hline & & & Against-direction & $280.21(33.69)$ & $275.84(45.50)$ & $>0.99$ \\
\hline & & & & $>0.99$ & $>0.99$ & \\
\hline
\end{tabular}




\subsection{Effects of habitual reading direction on antisaccade eye}

\section{movements} antisaccade latency for the correct trials $(\mathrm{F}(1,60)<0.82, \mathrm{p}>0.37$; Table 2$)$.

217 stimulus positions, stimulus magnitude (i.e., $5^{\circ}$ vs. $10^{\circ}$ ) was found to significantly affect the rate of directional errors $(\mathrm{F}(1,60)=9.25, \mathrm{p}=0.004)$, that both groups made more antisaccade errors towards $5^{\circ}$ targets compared with $10^{\circ}$ targets (rate of 0.24 vs. 0.17 and 0.24 vs. 0.10 for the LTR and RTL group respectively; Fig. 2). A significant interaction between group and stimulus direction (i.e., with- vs. against-reading direction) was observed $(F(1,60)=6.92$, $\mathrm{p}=0.01$ ), that the LTR group marginally had more antisaccade errors for targets appearing $10^{\circ}$ away from the centre at their habitual reading side, compared with the RTL group (rate of 0.22 vs. $0.07, \mathrm{p}=0.05)$.

Fig. 2 Antisaccade error rate in 2 groups of participants

The antisaccade error rate of the Chinese (left panel) as well as the Arabic and Persian group (right panel) in responding to the $5^{\circ}$ and $10^{\circ}$ with-direction target was $0.25 \pm 0.18$ and target was $0.23 \pm 0.21$ and $0.12 \pm 0.15$, and $0.27 \pm 0.21$ and $0.14 \pm 0.15$ respectively.

230 Bars are mean value and standard deviation.

\section{$231 \quad 3.3 \quad$ Relationship between prosaccade and antisaccade}


to have shorter prosaccade (174.42 vs. $188.06 \mathrm{msec})$ but longer antisaccade latency $(284.75$ reach significance $(F(1,60)=3.74, p=0.06)$ (see Fig. 3 ).

Fig. 3 Prosaccade latency and correct antisaccade latency of Chinese (LTR) as well as

Mean prosaccade latency for the Chinese as well as the Arabic and Persian groups was

\subsection{Effects of habitual reading direction on self-paced saccadic}

\section{eye movements}

Inter-saccadic interval and gain was compared between groups and directions of selfpaced saccades (i.e., saccades towards habitual reading direction vs. towards non-habitual reading direction) as well as the interaction effect. No significant group or direction effect was found on interval $(\mathrm{F}(1,30)<0.14, \mathrm{p}>0.71$; mean of 509.77 vs. $514.57 \mathrm{msec}$ in the Chinese group and 512.55 vs. $514.29 \mathrm{msec}$ in the Arabic and Persian group for saccades made towards habitual and non-habitual reading direction respectively).

However, a significant interaction between group and saccadic direction was observed on self-paced saccade gain $(F(1,30)=14.37, p<0.001$; see Fig. 4). The Chinese group showed more accurate gain when they made a saccade to the dot located at the side of their habitual reading direction (i.e., the dot at the right of the monitor) compared with the dot located in their non-habitual reading direction (mean 1.01 vs. $0.95, \mathrm{p}=0.02$ ). Whereas participants in the

256 Arabic and Persian group generated more accurate saccades towards the dot showing along 
Fig. 4 Self-paced saccade gain in Chinese (LTR) as well as the Arabic and Persian group (RTL)

The mean of self-paced saccade gain for the Chinese as well as the Arabic and Persian groups made towards the dot showing at their habitual reading direction was 1.01 and 0.96 respectively, while that made towards the dot located at the side of their non-habitual reading direction was 0.95 and 1.01 respectively.

$*: p<0.05$

\section{Discussion}

The objective of this study was to evaluate the impact of the primary habitual reading direction on the directionality of saccadic eye movements to low-cognitive-demand stimuli in young and healthy Chinese as well as Arabic and Persian participants using prosaccade, from left to right (LTR) in the prosaccade task compared with that of the Arabic and Persian participants whose primary habitual reading direction was from right to left (RTL). However, the effect of reading direction on the antisaccade latency disappeared, where participants in both groups had similar latencies of accurate antisaccade. The second major finding was that the Chinese subjects generated marginally but significantly more directional errors compared with the Arabic and Persian subjects when the target appeared at $10^{\circ}$ along their habitual

278 reading direction in the antisaccade task.

\subsection{Impact of habitual reading direction on prosaccade latency}

In this study, we hypothesized that participants would produce shorter prosaccade latency to a stimulus which appeared in their non-habitual reading direction (i.e., left for the

282 Chinese participants and right for the Arabic and Persian participants). This was, however, 
only found in the Arabic and Persian participants in responding to the $5^{\circ}$ target. Previous studies reported that the direction of the stimulus presentation did not significantly affect the prosaccade latency of young participants, although previous studies did not consider the participants' reading direction [41, 49]. In addition, our study found that Chinese readers had $16 \%$ shorter prosaccade latency than Arabic and Persian readers when target appeared $5^{\circ}$ along their habitual reading direction. Amatya et al. reported that Chinese participants generated more low latency 'express saccades' compared to non-Chinese participants (Caucasian participants) in an overlap prosaccade task [50]. Their study argued that this difference in saccade latency should be attributed to human genetic diversity rather than cultural differences, as those Chinese participants who grew up in the UK also showed the same pattern of saccade latency as the participants lived in mainland China [51]. A similar study by Knox and colleagues evaluated the antisaccade performance of Chinese participants and found a significantly higher antisaccade directional error rate in those Chinese participants who exhibited a higher proportion of express saccades [52]. They suggested that

297 there was a difference in neurophysiological substrate concerned with eye movement that

298 was not associated with culture. Nevertheless, in addition to express saccade latency, few studies have demonstrated the difference in normal reflexive saccades with both top-down and bottom-up control between populations. An electrophysiological study in primates showed that a neural signal took around $40 \mathrm{msec}$ to be transmitted from the retina to the

302 superior colliculus (SC), and it took approximately $20 \mathrm{msec}$ to stimulate the SC to trigger a

303 saccadic eye movement to a specific location [53]. However, the typical latency of a

304 prosaccade is around $200 \mathrm{msec}$ in humans [8]. Carpenter argued that such a long latency of

305 the saccadic eye movement was due to the decision time on making a decision to look at the 306 target or not [54]. One possible explanation for the different reaction time across groups was 307 that these two groups' participants used different decision-making strategies, resulting in 
different decision-making times. However, further study is required to investigate the decision-making time for simple cognitive tasks between different populations.

It has been suggested that attention needs to orient to the target location prior to the execution of the saccade [20]. Pollatsek and colleagues measured the perceptual span in bilingual Israeli readers who spoke English as their second language. They found that bilingual Israeli readers showed an asymmetry perceptual span that extended 14 characters to the left of fixation and 4 characters to the right while reading Hebrew [55]. Additionally, although no overall extent of the perceptual span was examined, Jorden et al., reported a leftward asymmetry in perceptual span when participants read Arabic [56]. McConkie and Rayner reported that skilled readers of English and other alphabetic languages reading from left to right showed an asymmetric perceptual span, extending 14-15 characteristics to the right of fixation and 3-4 characteristics to the left [57]. In contrast, Chinese readers showed a narrower perceptual span that extended 1 character space leftward and 3 characters spaces rightward as reported in Inhoff and Liu [58] or extended beyond 4 characters spaces rightward depending on the font size as reported in Yan et al. [59]. It is possible that the early disengagement of attention in Chinese participants leads to a reduction in prosaccade latency because the $5^{\circ}$ targets in the present study exceed the perceptual span used to acquire useful information by Chinese participants (the size of each character in [58] study was $0.9^{\circ}$ ), but still fall into the perceptual span of the Arabic and Persian participants. Accordingly, the Arabic and Persian group showed a directional difference of prosaccade latency whereas no group difference was found for the $10^{\circ}$ target away from the centre.

\subsection{Impact of habitual reading direction on prosaccade gain}

Inconsistent with other studies [44, 60, 61], we did not find a significant gain difference with respect to stimulus magnitude. One possible explanation could be the different perceptual span of the 2 groups of the participants. Therefore, hypometric 
performance towards a $5^{\circ}$ target disappeared when we combined the 2 groups together. A

334 significant interaction between group and stimulus direction was found in prosaccade gain.

335 Although Arabic and Persian subjects made more accurate prosaccade towards the side of

336 their non-habitual reading direction (i.e., right side of the fixation), Chinese participants also

337 performed similarly that target at the right side elicited more accurate prosaccades. This

338 finding was consistent with the result as reported in (Vergilino-Perez et al., 2012) that

339 rightward prosaccade had larger amplitude. Nevertheless, no primary habitual reading

340 direction effect was found on prosaccade gain.

\subsection{Impact of habitual reading direction on antisaccade latency}

The current result agreed with previous findings where the latency of a correct

343 horizontal antisaccade was independent of stimulus direction or magnitude [44, 62, 63].

344 Although different eye movement paradigms (overlap and gap conditions) were tested on different participant cohorts, the latency of the correct antisaccades did not show the same pattern as the prosaccades. The study reported by Knox et al. also revealed that the correct antisaccade latency was identical between Chinese participants who exhibited a high proportion of express saccades and those who did not [52]. Reading relies more on perceptually driven saccades [64]. In contrast, cognition is needed to inhibit the reflexive error that was stimulated by a perceptual stimulus in the antisaccade task [8]. Therefore, the possibility that the cognitive difference induced by subjects' habitual reading habits had greater influence on reflexive saccades compared to volitional saccades. That is, reaction time of a reflexive saccade was more impacted by reading direction than initiation time of an antisaccade. Therefore, the correct antisaccade latency was not significantly affected by the habitual reading direction or the direction of the stimulus. 


\subsection{Impact of habitual reading direction on antisaccade error} although shorter prosaccade latency was observed in the Chinese group when the target appeared $5^{\circ}$ along their habitual reading direction. Previous study showed that prosaccade training increased the number of antisaccade errors, because the reinforcement of the practice made it harder to inhibit a reflexive glance. Accordingly, subjects who were trained on

Persian participants in the present study were familiar with reading in both directions, the inhibition of reading in the opposite direction during one language processing might improve their ability to supress reflexive saccades, which resulted in significantly fewer number of errors. Although Arabic and Persian participants had shorter prosaccade latency towards their non-habitual reading direction side, they did not show higher antisaccade error rate, or more antisaccade errors towards the right side. This implies that this quicker prosaccade latency

371 was not fast enough to make more antisaccade errors.

\subsection{Impact of habitual reading direction on self-paced saccades}

The self-paced saccade task has been considered as an almost entirely volitional eye movement task as no reflexive cues are presented to trigger saccadic eye movements [9]. The generation of self-paced saccades requires a series of quick volitional engagements and disengagements of attention between 2 static stimuli. Although it has been proposed that

377 language processing drives the disengagement and shift of attention to the next word of 378 interest in the direction of reading [66], the current result failed to find a difference in the mean inter-saccadic interval between the self-paced saccades initiated to the side of subjects' 
habitual reading direction and those to the non-habitual reading direction in both groups. One was not shown in the current ocular motor task. Alternatively, it is possible that the subjects' sustained task engagement was more relevant to the performance of the self-paced saccade task, compared to the attentional modulation, as a result of the need to continuously initiate and execute eye movements [67]. Therefore, inter-saccadic interval was not significantly different between groups. However, both groups' participants showed more accurate gain when they made a self-paced saccade towards the right target. This result was similar with the prosaccades, where participants had more accurate gain when the target appeared at the right side.

\subsection{Limitations of the study}

At present, very few studies have investigated the differences in saccadic eye target supported our hypothesis, Nevertheless, we would like to point out several limitations uncertainty about the impact of habitual reading direction on saccadic eye movements as the

401 Arabic and Persian participants in the present study were experienced in reading both

402 directions. Secondly, we did not recruit participants in addition to Chinese who habitually 
habit differences. Finally, the current study had a relatively small sample size, limiting the performed on a large-scale cohort.

\section{Conclusions}

In the current study, we aimed to find the effect of the primary habitual reading

411 direction on the directionality of the characteristics of saccadic eye movements in healthy

412 Chinese as well as Arabic and Persian participants using prosaccade, antisaccade and self-

413 paced tasks. We hypothesised that participants showed shorter prosaccade latency and a

414 higher antisaccade error rate when a stimulus was presented at the side of their non-habitual

415 reading direction. Our hypotheses were partially accepted, with significantly shorter

416 prosaccade latency found in the Arabic and Persian participants in responding to the $5^{\circ}$

417 rightward target. The present study may contribute to the investigation of the neural

418 mechanisms of oculomotor behaviours between populations.

\section{Declarations of interest: none.}

\section{References}

421 1. Carpenter RHS. The neural control of looking. Curr Biol. 2000;10(8):R291-3.

422 2. Irwin DE. Information integration across saccadic eye movements. Cogn Psychol.

$423 \quad 1991 ; 23(3): 420-56$.

424 3. Pretegiani E, Optican LM. Eye Movements in Parkinson's Disease and Inherited

425 Parkinsonian Syndromes. Front Neurol. 2017;8:592.

426 4. Carpenter RHS. Frontal cortex. Choosing where to look. Curr Biol. 1994;4(4):341-3.

427 5. Hutton SB, Ettinger U. The antisaccade task as a research tool in psychopathology: a

428 critical review. Psychophysiology. 2006;43(3):302-13. 
429 6. Massen C. Parallel programming of exogenous and endogenous components in the 430 antisaccade task. Q J Exp Psychol A. 2004;57(3):475-98.

431 7. Munoz DP, Everling S. Look away: the anti-saccade task and the voluntary control of 432 eye movement. Nat Rev Neurosci. 2004;5(3):218-28.

433 8. Hutton SB. Cognitive control of saccadic eye movements. Brain Cogn. $434 \quad 2008 ; 68(3): 327-40$.

435 9. Abel LA, Douglas J. Effects of age on latency and error generation in internally 436 mediated saccades. Neurobiol Aging. 2007;28(4):627-37

437 10. Bittencourt J, Velasques B, Teixeira S, Basile LF, Salles JI, Nardi AE, et al. Saccadic 438 eye movement applications for psychiatric disorders. Neuropsychiatr Dis Treat. 2013;9:1393439409.

440 11. Broerse A, Crawford TJ, den Boer JA. Parsing cognition in schizophrenia using 441 saccadic eye movements: a selective overview. Neuropsychologia. 2001;39(7):742-56.

442 12. Barkley RA. Behavioral inhibition, sustained attention, and executive functions: 443 constructing a unifying theory of ADHD. Psychol Bull. 1997;121(1):65-94.

444 13. Gould TD, Bastain TM, Israel ME, Hommer DW, Castellanos FX. Altered 445 performance on an ocular fixation task in attention-deficit/hyperactivity disorder. Biol 446 Psychiatry. 2001;50(8):633-5.

447 14. Feifel D, Farber RH, Clementz BA, Perry W, Anllo-Vento L. Inhibitory deficits in 448 ocular motor behavior in adults with attention-deficit/hyperactivity disorder. Biol Psychiatry. $449 \quad 2004 ; 56(5): 333-9$.

450 15. Lezak MD. Neuropsychological assessment: Oxford University Press, USA; 2004.

451 16. Lemos J, Pereira D, Almendra L, Rebelo D, Patrício M, Castelhano J, et al. Distinct 452 functional properties of the vertical and horizontal saccadic network in Health and 453 Parkinson's disease: An eye-tracking and fMRI study. Brain Res. 2016;1648(Pt A):469-84. 
454 17. Winograd-Gurvich C, Georgiou-Karistianis N, Fitzgerald PB, Millist L, White OB.

455 Ocular motor differences between melancholic and non-melancholic depression. J Affect

456 Disord. 2006;93(1-3):193-203.

457 18. Fischer B, Weber H. Effects of stimulus conditions on the performance of

458 antisaccades in man. Exp Brain Res. 1997;116(2):191-200.

459 19. Liversedge SP, Findlay JM. Saccadic eye movements and cognition. Trends Cogn

460 Sci. 2000;4(1):6-14.

461 20. Hoffman JE, Subramaniam B. The role of visual attention in saccadic eye movements.

462 Percept Psychophys. 1995;57(6):787-95.

463 21. Roberts RJ, Hager LD, Heron C. Prefrontal cognitive processes: Working memory 464 and inhibition in the antisaccade task. J Exp Psychol Gen. 1994;123(4):374.

465 22. Dick S, Kathmann N, Ostendorf F, Ploner CJ. Differential effects of target probability

466 on saccade latencies in gap and warning tasks. Exp Brain Res. 2005;164(4):458-63.

467 23. Saslow MG. Effects of components of displacement-step stimuli upon latency for

468 saccadic eye movement. J Opt Soc Am. 1967;57(8):1024-9.

469 24. Carpenter RHS, Williams ML. Neural computation of log likelihood in control of $470 \quad$ saccadic eye movements. Nature. 1995;377(6544):59-62.

471 25. Inhoff AW, Pollatsek A, Posner MI, Rayner K. Covert attention and eye movements

472 during reading. Q J Exp Psychol A. 1989;41(1):63-89.

473 26. Han S, Northoff G. Culture-sensitive neural substrates of human cognition: A

474 transcultural neuroimaging approach. Nat Rev Neurosci. 2008;9(8):646.

475 27. Han S, Northoff G. Reading direction and culture. Nat Rev Neurosci. 2008;9(12):965.

476 28. Vaid J, Singh M. Asymmetries in the perception of facial affect: is there an influence

477 of reading habits? Neuropsychologia. 1989;27(10):1277-87. 
29. Chokron S, De Agostini M. Reading habits influence aesthetic preference. Brain Res

479 Cogn Brain Res. 2000;10(1-2):45-9.

480 30. Chokron S, Imbert M. Influence of reading habits on line bisection. Brain Res Cogn

$481 \quad$ Brain Res. 1993;1(4):219-22.

482 31. Eviatar Z. Reading direction and attention: effects on lateralized ignoring. Brain 483 Cogn. 1995;29(2):137-50.

484 32. Kazandjian S, Chokron S. Paying attention to reading direction. Nat Rev Neurosci. 2008;9(12):965-5.

486 33. Rinaldi L, Di Luca S, Henik A, Girelli L. Reading direction shifts visuospatial 487 attention: an Interactive Account of attentional biases. Acta Psychol (Amst). 2014;151:98488105.

34. Afsari Z, Ossandón JP, König P. The dynamic effect of reading direction habit on spatial asymmetry of image perception. J Vis. 2016;16(11):8.

$49135 . \quad$ Rayner K. Eye guidance in reading: fixation locations within words. Perception. $492 \quad 1979 ; 8(1): 21-30$.

493 36. Nuthmann A, Engbert R, Kliegl R. Mislocated fixations during reading and the 494 inverted optimal viewing position effect. Vision Res. 2005;45(17):2201-17.

495 37. Deutsch A, Rayner K. Initial fixation location effects in reading Hebrew words. Lang 496 Cogn Process. 1999;14(4):393-421.

497 38. Yan M, Zhou W, Shu H, Yusupu R, Miao D, Krügel A, et al. Eye movements guided 498 by morphological structure: evidence from the Uighur language. Cognition. 2014;132(2):181499215.

500 39. Yan M, Pan J, Chang W, Kliegl R. Read sideways or not: Vertical saccade advantage 501 in sentence reading. Read Writ. 2019;32(8):1911-26. 
40. Beydagi H, Yilmaz A, Süer C. The effect of direction on saccadic eye movement parameters. J Basic Clin Physiol Pharmacol. 1999;10(1):73-7.

504 41. Constantinidis TS, Smyrnis N, Evdokimidis I, Stefanis NC, Avramopoulos D,

505 Giouzelis I, et al. Effects of direction on saccadic performance in relation to lateral

506 preferences. Exp Brain Res. 2003;150(4):443-8.

507 42. Tagu J, Doré-Mazars K, Lemoine-Lardennois C, Vergilino-Perez D. How Eye

508 Dominance Strength Modulates the Influence of a Distractor on Saccade Accuracy. Invest

509 Ophthalmol Vis Sci. 2016;57(2):534-43.

510 43. Killgore WD, Yurgelun-Todd DA. Activation of the amygdala and anterior cingulate

511 during nonconscious processing of sad versus happy faces. Neuroimage. 2004;21(4):1215-23.

512 44. Vergilino-Perez D, Fayel A, Lemoine C, Senot P, Vergne J, Doré-Mazars K. Are

513 there any left-right asymmetries in saccade parameters? Examination of latency, gain, and

514 peak velocity. Invest Ophthalmol Vis Sci. 2012;53(7):3340-8.

515 45. Irving EL, Steinbach MJ, Lillakas L, Babu RJ, Hutchings N. Horizontal saccade

516 dynamics across the human life span. Invest Ophthalmol Vis Sci. 2006;47(6):2478-84.

517 46. Fischer B, Breitmeyer B. Mechanisms of visual attention revealed by saccadic eye 518 movements. Neuropsychologia. 1987;25(1A):73-83.

519 47. Fischer B. The preparation of visually guided saccades. Rev Physiol Biochem

520 Pharmacol. 1987;106:1-35.

521 48. Fischer B, Ramsperger E. Human express saccades: extremely short reaction times of 522 goal directed eye movements. Exp Brain Res. 1984;57(1):191-5.

523 49. De Clerck M, Crevits L, Van Maele G. Saccades: is there a difference between right 524 and left? Neuroophthalmology. 2000;24(2):327-30.

525 50. Amatya N, Gong Q, Knox PC. Differing proportions of 'express saccade makers' in 526 different human populations. Exp Brain Res. 2011;210(1):117-29. 
527 51. Knox PC, Wolohan FD. Cultural diversity and saccade similarities: culture does not

528 explain saccade latency differences between Chinese and Caucasian participants. PLoS One.

530 52. Knox PC, Amatya N, Jiang X, Gong Q. Performance deficits in a voluntary saccade 531 task in Chinese "express saccade makers". PLoS One. 2012;7(10):e47688.

532 53. Carpenter RHS. Oculomotor procrastination. Eye movements: cognition and visual perception. Fischer DF, Monty RA, eds. 1981. pp237-46.

534 54. Carpenter RHS. Express saccades: is bimodality a result of the order of stimulus 535 presentation? Vision Res. 2001;41(9):1145-51.

536 55. Pollatsek A, Bolozky S, Well AD, Rayner K. Asymmetries in the perceptual span for 537 Israeli readers. Brain Lang. 1981;14(1):174-80.

538 56. Jordan TR, Almabruk AAA, Gadalla EA, McGowan VA, White SJ, Abedipour L, et

539 al. Reading direction and the central perceptual span: Evidence from Arabic and English.

$540 \quad$ Psychon Bull Rev. 2014;21(2):505-11.

541 57. McConkie GW, Rayner K. The span of the effective stimulus during a fixation in 542 reading. Percept Psychophys. 1975;17(6):578-86.

543 58. Inhoff AW, Liu W. The perceptual span and oculomotor activity during the reading of

544 Chinese sentences. J Exp Psychol Hum Percept Perform. 1998;24(1):20-34.

545 59. Yan M, Zhou W, Shu H, Kliegl R. Perceptual span depends on font size during the 546 reading of Chinese sentences. J Exp Psychol Learn Mem Cogn. 2015;41(1):209-19.

547 60. Ploner CJ, Ostendorf F, Dick S. Target size modulates saccadic eye movements in 548 humans. Behav Neurosci. 2004;118(1):237-42.

549 61. Kapoula Z. Evidence for a range effect in the saccadic system. Vision Res. $550 \quad 1985 ; 25(8): 1155-7$. 
551 62. Bell AH, Everling S, Munoz DP. Influence of stimulus eccentricity and direction on

552 characteristics of pro- and antisaccades in non-human primates. J Neurophysiol.

$553 \quad 2000 ; 84(5): 2595-604$.

554 63. Fischer B, Biscaldi M, Gezeck S. On the development of voluntary and reflexive

555 components in human saccade generation. Brain Res. 1997;754(1):285-97.

556 64. Feng G. Is there a common control mechanism for anti-saccades and reading eye

557 movements? Evidence from distributional analyses. Vision Res. 2012;57:35-50.

558 65. Dyckman KA, McDowell JE. Behavioral plasticity of antisaccade performance

559 following daily practice. Exp Brain Res. 2005;162(1):63-9.

560 66. Morrison RE. Manipulation of stimulus onset delay in reading: evidence for parallel

561 programming of saccades. J Exp Psychol Hum Percept Perform. 1984;10(5):667.

562 67. Azizi E. The influence of playing video games as an attention rehabilitation technique

563 in patients with traumatic brain injury. Published PhD thesis. Unoversity of Melbourne. 2016. 


\section{Calibration}

Fig. 1a

\section{Fixation}

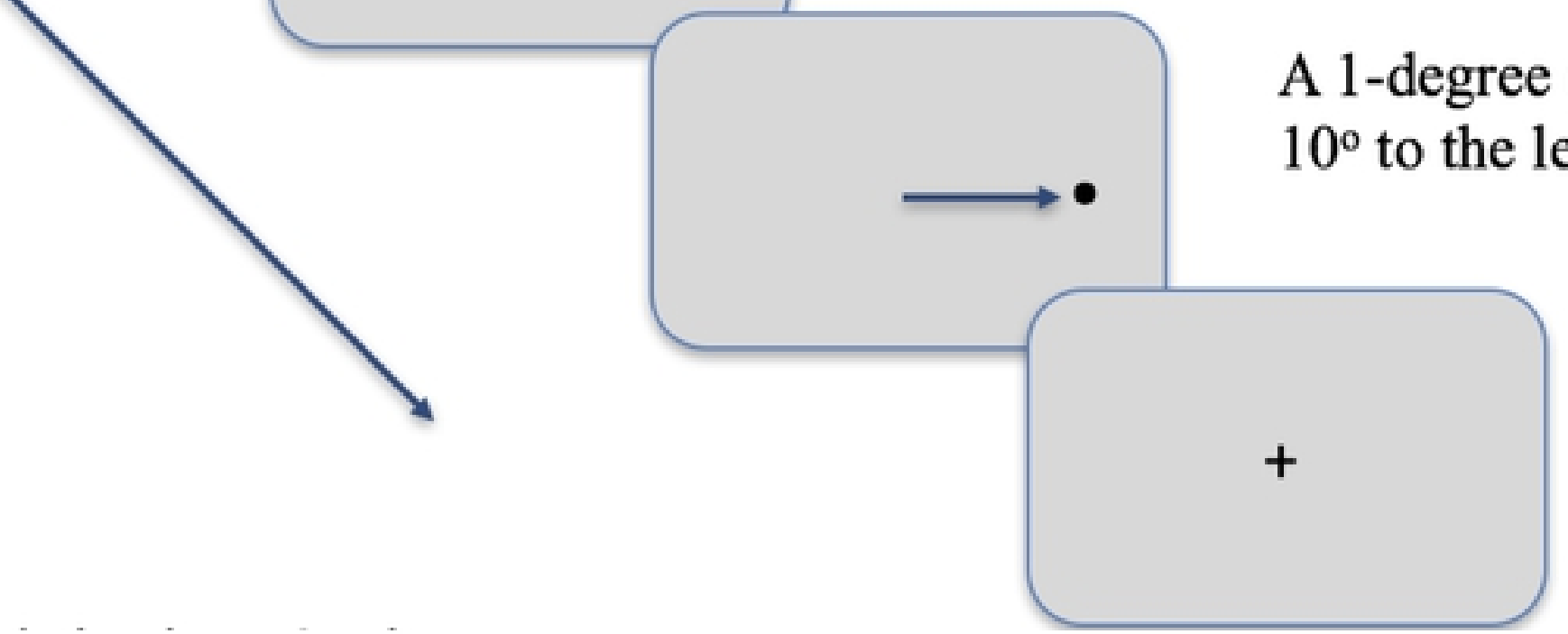

Inter-stimulus interval ranged from $1000 \mathrm{~ms}$ to $2000 \mathrm{~ms}$. 
Calibration

\section{Fixation}

$+$

A 1-degree dot randomly appeared at $5^{\circ}$ or $10^{\circ}$ to the left or right of the center.

Inter-stimulus interval ranged from $1000 \mathrm{~ms}$ to $2000 \mathrm{~ms}$

Fig. $1 \mathrm{~b}$ 
Antisaccade error rate in Chinese (LTR) group

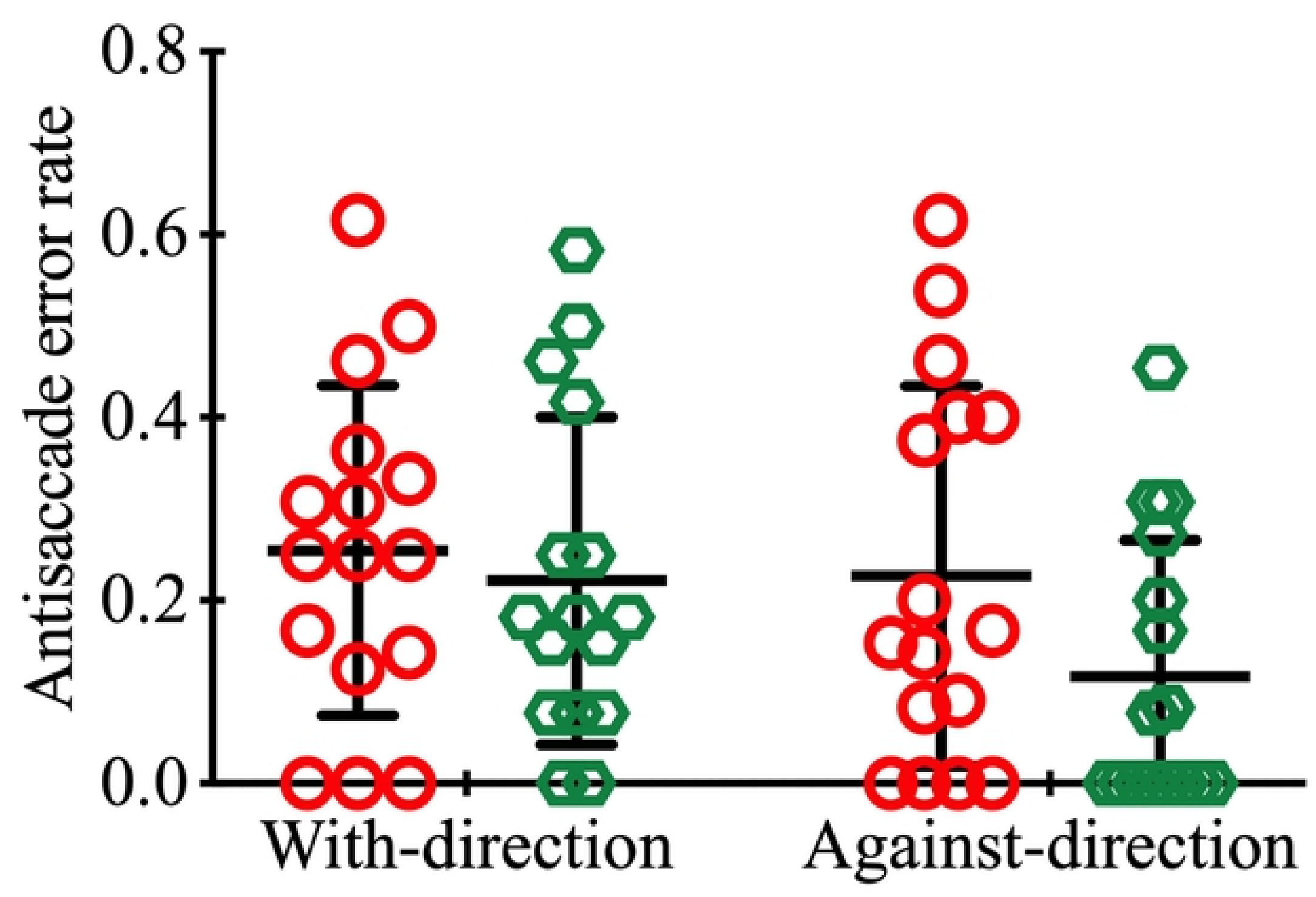

O $5^{\circ}$ target

- $10^{\circ}$ target

Stimulus direction

Fig. 2_left 
Antisaccade error rate in Arabic and Persian (RTL) group

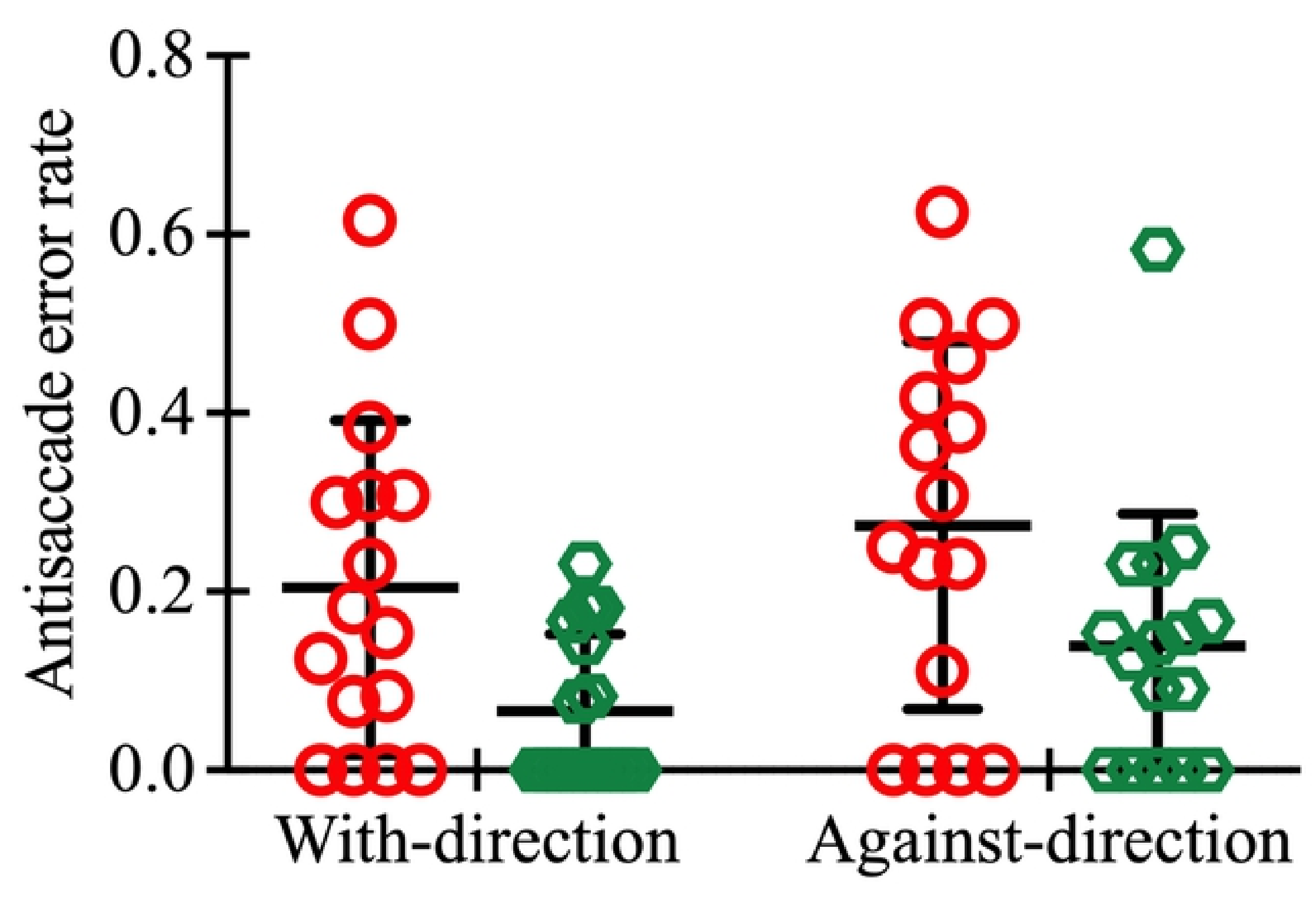

Stimulus direction

Fig. 2_right 



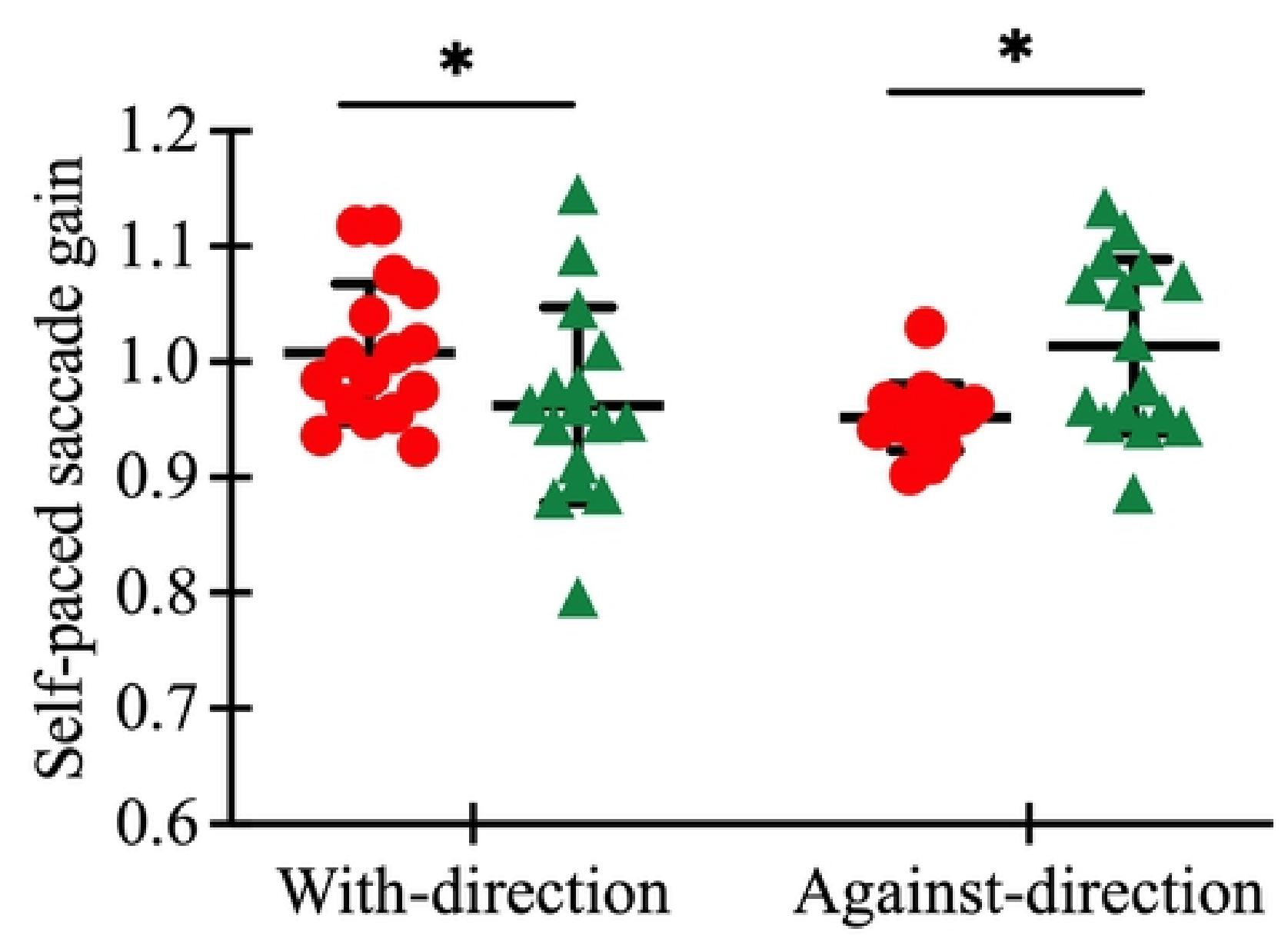

- Chinese participants

A Arabic and Persian participants

Direction of self-paced saccade

Fig. 4 